more patients in the condom-catheter group reported that their catheter was comfortable and not painful (both $P=0.02$ ) than patients in the indwelling-catheter group.

The authors caution that these results only apply to the specific condom catheter used in this study: this newly developed catheter is less prone to be dislodged than earlier models. It is also important to note that dementia was three times more common in the condom-catheter group. Nevertheless, the authors recommend that condom catheters be used in preference to indwelling catheters, wherever possible.

Original article Saint S et al. (2006) Condom versus indwelling urinary catheters: a randomized trial. J Am Geriatr Soc 54: 1055-1061

\section{Pediatric detrusor overactivity can be treated with botulinum $A$ toxin}

Botulinum type A toxin (BTX-A) has been successfully used to treat detrusor overactivity in adults, but its efficacy in children is unknown. Its safety in the treatment of other pediatric disorders, however, has been proven. As detrusor overactivity is common in children, Hoebeke et al. tested the effectiveness of BTX-A in the treatment of pediatric, therapy-resistant, non-neurogenic detrusor overactivity.

In total, 21 children (mean age 10.8 years) participated in the 19-month trial; all received 100 U BTX-A injected into 15 detrusor-muscle sites (1 $\mathrm{ml}$ per site). One girl experienced 10-day temporary urinary retention, two girls contracted lower urinary tract infections, and one boy had temporary vesicoureteral reflux. Of 15 children who were followed up for $\geq 6$ months, nine showed a complete response (defined as no more urge and dry during the day), three showed a partial response (decreased urge and incontinence), and three showed no response. One of the nine children who showed a complete response relapsed after 8 months, but the remaining eight were still cured at 12 months. The three children who showed a partial response and the child who relapsed were given a second BTX-A treatment; this resulted in two complete responses.

Although these results are encouraging, it should be remembered that overactive bladder is a more dynamic condition in children than it is in adults, and treatment strategies should be decided with this in mind. Hoebeke et al. note that current BTX-A doses are somewhat arbitrary, and recommend that the optimal clinical dose be investigated.

Original article Hoebeke P et al. (2006) The effect of botulinum-A toxin in incontinent children with therapy resistant overactive detrusor. J Urol 176: 328-331

\section{Does Viagra ${ }^{\circledR}$ improve lower urinary tract symptoms in men with erectile dysfunction?}

Previous studies have suggested a physiologic link between lower urinary tract symptoms (LUTS) secondary to benign prostatic hyperplasia, and erectile dysfunction. In addition, anecdotal evidence exists for a beneficial effect of the phosphodiesterase-5-inhibitor sildenafil citrate (Viagra ${ }^{\circledR}$, Pfizer, NY) on LUTS.

In this noncontrolled, nonrandomized study, Mulhall and colleagues enrolled 48 Viagra $^{\circledR}$-naive men (mean age $62 \pm 11$ years) with erectile dysfunction, who scored $>10$ on the International Prostate Symptom Score (IPSS) at baseline. Men completed both the International Index of Erectile Function (IIEF) and the IPSS questionnaires before, and at least 3 months after, the start of 'as-needed' Viagra $^{\circledR}$ therapy.

After treatment (mean 2 uses per week), a marked improvement was seen in the mean health-related quality-of-life and in the IEFF erectile-dysfunction domain score. IPSS markedly improved after treatment in 60\% of participants. The degree of IPSS improvement was not related to baseline IPSS, or to change in or baseline erectile-function score, however, which the authors suggest might reflect differences between the severity of LUTS and the severity of erectile dysfunction in individual patients.

The authors suggest that an improvement in erectile function might improve a patient's perceived quality of life, such that the severity of LUTS seems reduced. Viagra ${ }^{\circledR}$ might also reduce LUTS by inducing relaxation of smooth muscle of the prostate and/or bladder neck. A randomized, controlled trial of Viagra ${ }^{\circledR}$ in this setting, that uses objective measures of LUTS improvement, is underway.

Original article Mulhall JP et al. (2006) Assessment of the impact of sildenafil citrate on lower urinary tract symptoms in men with erectile dysfunction. J Sex Med 3: 662-667 